\title{
Norois
}

Environnement, aménagement, société

$224 \mid 2012$

Agriculture paysanne, circuits courts, territoires périurbains

\section{Les circuits courts, des innovations sociales pour une alimentation durable dans les territoires}

Short food supply chains as social innovations for sustainable food in territories

\section{Yuna Chiffoleau et Benoît Prevost}

\section{CpenEdition}

\section{Journals}

Édition électronique

URL : http://journals.openedition.org/norois/4245

DOI : $10.4000 /$ norois. 4245

ISBN : 978-2-7535-2157-5

ISSN : $1760-8546$

\section{Éditeur}

Presses universitaires de Rennes

\section{Édition imprimée}

Date de publication : 30 septembre 2012

Pagination : 7-20

ISBN : 978-2-7535-2155-1

ISSN : 0029-182X

\section{Référence électronique}

Yuna Chiffoleau et Benoît Prevost, "Les circuits courts, des innovations sociales pour une alimentation durable dans les territoires », Norois [En ligne], 224 | 2012, mis en ligne le 30 septembre 2014, consulté le 10 décembre 2020. URL : http://journals.openedition.org/norois/4245 ; DOI : https:// doi.org/10.4000/norois.4245 


\title{
Les circuits courts, des innovations sociales pour une alimentation durable dans les territoires
}

\author{
Short Food Supply Chains as Social Innovations \\ for Sustainable Food in Territories
}

Yuna Chiffoleau ${ }^{\mathrm{a} *}$, Benoît Prévost

* Auteur correspondant

a INRA, Centre de Recherche de Montpellier, Unité Mixte de Recherche Innovation et Développement -

34060 Montpellier Cedex 1

b Université Montpellier 3, UMR 5281 ARTDev-CNRS (benoit.prevost@univ-montp3.fr)

Résumé : Les circuits courts agro-alimentaires constituent des innovations territoriales participant d'une reformulation des dynamiques locales via l'émergence de pratiques socio-économiques dont les implications relèvent des différentes dimensions du développement durable. Ils participent notamment d'un empowerment non seulement des producteurs, mais aussi de l'ensemble des partenaires territoriaux autour de la mobilisation en faveur d'une alimentation durable.

Fondé sur les résultats de plusieurs projets, l'article propose de dépasser la notion d'innovation sociale au profit de celle d'innovation territoriale, plus à même d'englober les mécanismes d'entraînement qui permettent à un territoire de générer des innovations socioéconomiques et à ses acteurs de s'en saisir. Il revient sur les implications théoriques de l'analyse des circuits courts, notamment sur l'articulation entre l'approche par les capabilités et la Nouvelle sociologie économique. Enfin, il montre les apports d'une telle articulation pour l'appréhension et la compréhension des dynamiques territoriales autour des circuits courts, dans la perspective d'une gouvernance territoriale pour une alimentation durable.

\begin{abstract}
Short food supply chains occur as territorial innovations taking part in the reformulation of local dynamics through the emergence of socio-economic practices whose implications pertain to the different dimensions of sustainable development. They take part in the empowerment not only of producers but also of the set of territorial partners around a mobilization in favour of sustainable food. Based on results from several projects, the paper proposes to go beyond the notion of social innovation for the benefit of territorial innovation, more able to encompass the driving mechanisms which allow a territory to generate socio-economic innovations and the actors to catch these ones. The paper goes back over the theoretical implications of short food supply chains analysis, especially over the link between the approach through capabilities and the New economic sociology. Finally, he highlights the contribution of such a link to seize and understand territorial dynamics around short food supply chains, in the perspective of a territorial governance for a sustainable food.
\end{abstract}

Mots clés : circuits courts - alimentation durable - innovations territoriales - démocratie participative

Keywords: short food chain - sustainable food - spatial inovation - territorial democracy 


\section{INTRODUCTION : INNOVATIONS SOCIALES ET CIRCUITS COURTS}

Bien que leur définition ne soit pas stabilisée, on considère en règle générale que les innovations sociales concernent l'émergence de nouvelles modalités de coordination de relations entre les acteurs sociaux afin de répondre à des attentes sociales (Harrison et Vezina, 2006; Bouchard, 2006). Ces innovations constituent des alternatives aux mécanismes de coordination marchands, lorsque ceux-ci ne satisfont pas ou plus certains besoins liés au bien-être des populations. Dans cette perspective, la nécessité d'alternatives est liée aussi bien aux défaillances de marché que les économistes analysent depuis le $\mathrm{XIX}^{\mathrm{e}}$ siècle, qu'aux processus de désengagement de l'État. C'est ainsi, par exemple, que Nussbaumer et Moulaert (2008) replacent le succès des innovations sociales dans un contexte de privatisation néolibérale à partir des années 1990. C'est ainsi, également, que se tissent les ponts avec l'économie sociale et solidaire en tant que champ d'activités qui ne relèverait ni du marchand, ni du public, mais d'espaces d'hybridation entre diverses modalités de coordination, fondant alors l'économie sociale et solidaire également comme une discipline à même d'appréhender cette complexité (Laville, 2006).

Ces approches de l'innovation sociale privilégient le fait que celle-ci « se développe soit dans le cadre d'activités délaissées par le marché ou l'État, laissant supposer des interstices comme espaces d'innovations, soit en partenariat avec l'État et les collectivités territoriales » (Richez-Battesti et Vallade, 2009). Si cette perspective est souvent pertinente, elle risque de laisser croire à une séparation nette entre innovations sociales et marché alors que de nombreuses innovations sociales peuvent s'inscrire à la fois dans et contre le marché : elles peuvent avoir une dimension économique et marchande forte sans pour autant perdre leur nature dans la mesure où elles visent à modifier le fonctionnement de l'économie et du marché tel qu'il est, pour proposer d'autres modalités de fonctionnement, plus conformes aux attentes des acteurs socio-économiques (Prévost, 2011).

À partir de l'exemple des circuits courts alimentaires, définis comme des formes de vente mobilisant au plus un intermédiaire entre producteurs et consommateurs $^{1}$, nous voulons nous concentrer ici sur les innovations sociales qui génèrent des innovations territoriales, partant du principe que ces dernières reposent sur des effets d'entraînement liés aux innovations sociales. Des initiatives pour modifier la coordination marchande soutiennent ensuite de nouvelles modalités d'actions collectives concernant les relations entre les différentes catégories d'acteurs du développement territorial ${ }^{2}$. À partir de nombreux travaux de terrain ${ }^{3}$, l'enjeu est de rendre compte des impacts de ces circuits et des innovations territoriales associées sur l'émergence d'une alimentation durable au cœur de nouvelles conceptions du développement local dans les territoires. Concept non stabilisé également (Redlingshöfer, 2006), l'« alimentation durable » est entendue ici en tant qu'ensemble de pratiques, de la production à la consommation de biens alimentaires, économiquement viables, socialement soutenables et écologiquement responsables.

Nous proposons dans un premier temps un détour par l'analyse en termes de capabilités pour montrer en quoi elle permet d'articuler plusieurs perspectives théoriques issues de la "nouvelle sociologie économique » tout en rendant compte des nouvelles orientations en matière de stratégies de développement durable. Nous montrons ensuite comment la conception des choix individuels et de l'action collective qui en découle peut enrichir l'analyse des circuits courts en posant de manière originale des questions centrales identifiées par la recherche sur ces modes de commercialisation agro-alimentaire : d'une part la formation de prix susceptibles de satisfaire à la fois producteurs et consommateurs

1. Définition donnée par le Ministère de l'Agriculture, de l'Alimentation et de la Pêche (MAAP) en avril 2009, à partir d'un groupe de travail réunissant professionnels du secteur et représentants de la société civile. Au delà de la définition, le groupe a contribué à la construction d'un plan d'action pour le développement de ces circuits, dit «Plan Barnier».

2. Nous laissons ainsi de côté, les analyses liées à l'amélioration de la performance des firmes et organisations, qui sont une partie importante des innovations sociales, même si nous y reviendrons par d'autres biais. Nous ne nous intéressons donc pas ici aux relations internes aux différentes organisations qui peuvent être actrices de ce développement.

3. Les auteurs sont responsables de plusieurs projets de recherche (projet EQUAL-CROC, projet PSDR-Coxinel, étude pour le MAAP sur l'élaboration d'un référentiel technico-économique sur les Circuits Courts) et de l'animation du sous-groupe «Alimentation et agriculture » du Réseau Rural Français, contribuent au pilotage scientifique de plusieurs projets autour du développement des circuits courts (comme le Projet CASDARFédération Nationale des Parcs Naturels Régionaux), etc. Chacun des projets est basé sur des études de cas et des accompagnements d'initiatives dans la durée, combinant entretiens, enquêtes quantitatives et analyse des réseaux sociaux impliqués dans les dynamiques. Pour exemple, cf. Chiffoleau, 2009. 
et, d'autre part, l'évolution des attentes formulées par les différents acteurs impliqués dans les circuits courts autour du thème de l'alimentation durable. Nous concluons par les enjeux que représentent ces circuits au regard d'une gouvernance territoriale renouvelée, articulant les villes et les territoires les environnant à travers un processus démocratique d'évaluation du lien production-alimentation.

\section{Capabilités, participation DÉMOCRATIQUE ET CIRCUITS COURTS}

L'approche par les capabilités (Sen, 2003) ${ }^{4}$ est un terreau fertile à partir duquel de très nombreuses pistes de recherche se sont ouvertes dans les années 1990, en particulier pour l'analyse des phénomènes de pauvreté et de développement. Sen présente l'approche par les capabilités comme une alternative réelle à l'économie standard, en grande partie grâce à une représentation nouvelle des relations économiques, sociales et politiques s'appuyant sur un renouvellement ontologique et méthodologique. Cette représentation fait de la démocratie une valeur centrale et constitutive du développement. Ce positionnement intellectuel et moral est à la fois à la base et à l'achèvement du système de Sen; il en est aussi bien la fondation que la charnière centrale et l'objectif vers lequel tendent la plupart de ses démonstrations (Prévost, 2009). On peut ainsi considérer que la remise en cause des dogmes de l'orthodoxie n'a de sens que pour parvenir à cette finalité : montrer que la démocratie est indissociable du développement.

Cette conclusion des travaux de Sen repose sur le dépassement du paradoxe de Condorcet et du théorème d'impossibilité d'Arrow : elle trouve donc ses fondements dans une interrogation fondamentale sur l'émergence des choix collectifs, interrogation indissociable des problématiques de l'action collective qui est au cœur de l'innovation sociale (Prévost, 2011). C'est pourquoi l'approche par les capabilités peut trouver sa place dans les travaux sur l'innovation sociale en ouvrant de nouvelles pistes pour la compréhension de la manière dont les agents formulent

4. Sen (2003) est l'ouvrage le plus accessible pour une présentation de la théorie des capabilités. Pour des présentations synthétiques de ces apports, on pourra se référer à B. Prévost (2010), J. de Munck (2008) et E. Benicourt (2007) pour une critique qui a suscité la polémique. Dans la littérature anglo-saxonne, voir E. Anderson (2003), R. Putnam (2002), I. Robeyns (2005) et V. Walsh (2000, 2003). des choix individuels puis participent à des projets collectifs. En particulier, au contraire de la théorie standard enfermant les individus dans une série de préférences individuelles figées et pré-déterminées, Sen considère que les valeurs poursuivies par les individus (ce qu'ils souhaitent réaliser) sont le résultat d'une interaction sociale déterminant à la fois ce qu'il leur semble possible de réaliser ${ }^{5}$ et ce qu'il leur semble souhaitable de valoriser. La démocratie se définit alors comme « l'incarnation institutionnelle d'un raisonnement et d'une expérimentation collective quant aux modalités du vivre ensemble », ce qui en fait un « engagement collectif dans la raison pratique » qui dépasse l'action publique et inclut une participation aux débats des citoyens les plus ordinaires (Anderson, 2003). Cette pratique de la démocratie est indispensable à la connaissance des uns et des autres (Sen, 1999) et donc à la formation et à l'évolution des valeurs et des modes de vie que les individus peuvent souhaiter (Prévost, 2009) :

« La politique de la démocratie donne aux citoyens une chance d'apprendre les uns des autres et aide la société à donner forme à ses valeurs et à ses priorités. Même l'idée de «besoins », qui inclut la compréhension des besoins économiques, requiert une discussion publique et l'échange d'informations, de points de vue et d'analyses. Dans ce sens, la démocratie joue un rôle constructif. » (Sen, 2006, p. 71)

En d'autres termes, l'approche par les capabilités permet de comprendre comment la théorie peut saisir l'ouverture du champ des possibles qui est au cœur des processus d'innovation sociale.

\section{Implications pour le fonctionnement des marchés}

Les individus ne peuvent pas être analysés et appréhendés comme des agents autonomes disposant d'un ordre de préférence complet. Cette indétermination des préférences va à l'encontre de la démarche de l'économie standard, d'autant plus qu'elle implique également que les faits économiques et sociaux doivent être interprétés comme des processus complexes dans lesquels Parties et Tout forment un ensemble indissociable (Robeyns,

5. Il s’agit du problème des préférences adaptatives (par ex. A. Sen, 2001). 
2005). Si les comportements individuels restent la base de la compréhension des phénomènes collectifs, ils s'inscrivent dans un ensemble de relations sociales qui contribuent à les déterminer. Le prolongement logique de l'hypothèse suivant laquelle les préférences se construisent dans l'interaction sociale, en évitant les travers de la sur- et de la sousdétermination sociale, invite à un rapprochement avec la sociologie économique, renouvelée dans les années 80 suite à l'article clé de Granovetter sur l'encastrement (1985) et fondée sur les principes de l'interactionnisme structural (White, 1992, 2002; Granovetter, 2000).

Cela implique que les échanges doivent être analysés comme des relations sociales au cours desquelles se forment les préférences individuelles et non pas comme un simple moment de rencontre de ces préférences sous la forme d'une offre et d'une demande constituées par sommation d'utilités individuelles autonomes. Ces raisonnements sont d'autant plus importants sur des marchés marqués par l'incertitude, caractérisant l'économie de la qualité (Akerlof, 1970; Karpik, 1989) et illustrés en particulier par les marchés des biens alimentaires pour lesquels l'incertitude concerne tout aussi bien les consommateurs que les producteurs (Chiffoleau et Laporte, 2004). Les circuits courts alimentaires s'incrivent d'autant plus dans cette perspective qu'ils peuvent être considérés comme des formes d'échange qui privilégient un dialogue autour des produits dans une logique où la qualité des biens qui circulent dépend d'attentes complexes et partagées par les producteurs et les consommateurs.

De nombreux travaux sur la consommation montrent que les attentes des consommateurs en matière de produits alimentaires reposent sur une approche multidimensionnelle de la qualité : gustative, sanitaire, environnementale, sociale... (Codron et al., 2006). Si les circuits courts sont le lieu d'expression d'une « consommation engagée » valorisant les enjeux sociaux et/ou environnementaux (Dubuisson-Quellier et Lamine, 2006), ils ne se réduisent pas à cette dernière et sont aussi l'occasion d'une consommation beaucoup plus ordinaire. Une grille de lecture en termes d'engagement risque d'ailleurs de porter le débat sur des questions finalement marginales qui conduiraient à distinguer une consommation engagée d'une consommation purement égocentrée. Or l'analyse des comportements individuels doit se faire sur la base d'un continuum entre ces deux pôles (Pettit, 2004), les comportements de chacun évoluant en fonction des informations et des contraintes qui pèsent sur les choix, variant parfois d'un pôle à l'autre dans le cadre d'une société hypermoderne caractérisée par le «zapping » (Lipovetsky et Charles, 2004), soumise à l'influence des interactions et du contexte ad hoc. L'échange de biens alimentaires s'analyse donc comme l'élaboration d'attentes individuelles fondées sur l'échange d'informations entre producteurs et consommateurs, direct ou via un intermédiaire : informations sur la qualité des produits et sur les contraintes pesant sur la production et la consommation. Les circuits courts, dans leur diversité, peuvent alors être supposés constituer un ensemble d'innovations sociales qui permettent à la fois une circulation différente des biens et des informations mais aussi, et peut-être surtout, de nouveaux processus d'élaboration des attentes individuelles et des informations qui sont nécessaires à leur réalisation. Il peut s'agir en effet, pour les producteurs comme pour les consommateurs, d'une ouverture permanente des champs du possible, donc de leurs libertés individuelles.

Les circuits courts offrent aussi a priori une occasion importante pour l'analyse économique de repenser les mécanismes de formation des prix et de se pencher à nouveau sur les théories de la valeur : repenser les mécanismes de formation des prix car plusieurs systèmes de production et de commercialisation cohabitent et les réseaux associés à l'activité se complexifient (Chiffoleau et Laporte, 2004); se pencher à nouveau sur les théories de la valeur car la revendication croissante, au Nord, de prix agricoles équitables concerne aussi bien les producteurs qui aspirent à vivre de leur travail que les consommateurs qui aspirent à une alimentation de qualité. Ancrés dans des échanges locaux, certains circuits courts se distinguent alors en associant étroitement proximité, liens interpersonnels, transparence et confiance (Hinrichs, 2000; Lamine, 2005 ; Amemiya et al., 2008) : en ce cas, ils sont au cœur de la formation de prix dont l'acceptation relève d'une démarche participative dans laquelle les différentes parties prenantes peuvent exprimer ce qu'ils souhaitent valoriser à travers l'acte marchand. Nos travaux montrent que cet accord sur les prix entre produc- 
teurs et consommateurs n'est pas réservé au seul cas des $\mathrm{AMAP}^{6}$ : dans une commune périurbaine de Montpellier, par exemple, un marché de plein vent signale aux consommateurs trois origines de produits différentes à travers des codes couleurs. En vert, sont identifiés les produits des producteurs présents sur le marché, en orange, des produits achetés à d'autres producteurs bien identifiés ou fabriqués à partir des produits de ces derniers, et enfin en violet, sont proposés des produits à l'origine sociale et géographique plus lointaine mais « demandés par les consommateurs » (par exemple les carottes, très peu produites autour de Montpellier). Cette innovation sociale, issue d'une démarche participative impliquant l'ensemble des acteurs du territoire, permet finalement non seulement de signaler les marges liées à l'achat-revente mais aussi de les justifier et les faire accepter comme « justes » par les consommateurs dans le cas de l'orange, qui témoigne de l'entraide entre producteurs ou d'un partenariat entre producteur et revendeur ou artisan ${ }^{7}$. L'enjeu s'élargit même dans la mesure où le violet suscite de nouvelles interrogations chez certains consommateurs quant à ce qui est vraiment indispensable, entraînant pour quelques uns l'arrêt de leur achat au profit de produits en vert ou orange, ce qui renforce l'inscription de l'innovation territoriale dans le projet d'une alimentation durable. Accompagnée par notre équipe, cette innovation est aujourd'hui à l'étude dans d'autres territoires, mais risque de rester au stade d'invention sociale exogène si elle n'est pas repensée comme objet de démocratie locale.

\section{Implications pour l'action collective autour de l'alimentation durable}

La formation d'un prix juste suppose ainsi la prise en compte d'aspirations diverses, parfois contradictoires, qui renvoient plus largement aux critères à partir desquels évaluer la qualité des biens. Les débats sur les circuits courts, la diversité des travaux de recherche, les dialogues entre monde de la recherche et acteurs du développement agricole et rural (au sein du Réseau Rural Français notamment $^{8}$ ) montrent que, pour les acteurs concernés,

6. Association pour le Maintien d'une Agriculture Paysanne.

7. Pour en savoir plus, cf. [http://www.psdr-coxinel.fr], rubrique «Recherches».

8. Dispositif appliqué d'un règlement européen adressé à chaque Étatmembre, co-piloté en France par le MAAP et la DATAR, visant à fédérer les acteurs du monde agricole et rural pour capitaliser les bonnes pra- les externalités sont essentielles. Les circuits courts ne sont pas seulement une autre façon de faire circuler des biens standardisés : ils sont à la fois une alternative à la rationalisation industrielle et marchande des biens alimentaires (alternative stratégique pour les uns, contestataire pour d'autres) et une piste face aux questions du développement local. Une enquête nationale menée par notre équipe auprès d'acteurs impliqués dans le développement des circuits courts ${ }^{9}$ a permis notamment de montrer la grande diversité des enjeux associés, comme le montre le tableau 1.

L'idée que les circuits courts puissent répondre à une telle diversité d'attentes permet de saisir comment, progressivement, à partir de ces innovations sociales, le champ des possibles non seulement s'élargit mais aussi s'applique sur d'autres objets du territoire : si l'amélioration des revenus des agriculteurs demeure un objectif essentiel, il n'en reste pas moins que les acteurs du développement local articulent à cet objectif principal une série d'autres objectifs qui permettent de concevoir des projets globaux pour les territoires en faisant émerger à la fois de nouvelles attentes et de nouveaux moyens d'y parvenir. Cette modification des perspectives de développement local est importante lorsqu'on se focalise sur les questions relevant de l'alimentation durable.

La lecture des attentes par catégories d'acteurs, à travers cette enquête, révèle en effet une vision « élargie » de la production alimentaire distribuée en circuits courts car inscrite dans des préoccupations liées au développement de leur territoire, comme le montre le tableau 2.

Ces attentes peuvent de plus se croiser pour déboucher sur de nouvelles possibilités qui ne sont pas nécessairement envisagées au préalable. Ainsi, par exemple, comme le montrent les recherches de notre équipe et autres travaux sur ces circuits, les pratiques productives en circuits courts peuvent élargir la palette de choix des consommateurs au-delà de leurs attentes initiales (découverte de nouvelles variétés) et modifier leurs pratiques alimentaires (Merle et Piotrowski, 2012), y compris

\footnotetext{
tiques de développement territorial sur la période 2007-2013, cf. [http:// wow reseaurural.fr].

9. Enquête réalisée au niveau national auprès des associations de développement agricole et rural, conseils régionaux, généraux, chambres consulaires... Pour le détail des résultats obtenus : Voir Texeira M., 201 1, Rapport pour le Réseau rural français, [http://reseaurural.fr].
} 


\begin{tabular}{|l|l|}
\hline Aider les agriculteurs à améliorer leurs revenus & $66 \%$ \\
\hline Valoriser les ressources et le patrimoine local & $41 \%$ \\
\hline Sensibiliser les consommateurs aux enjeux d'une consommation plus éthique & $33 \%$ \\
\hline Adopter des pratiques agricoles plus durables & $33 \%$ \\
\hline Garantir l'accès à une alimentation de qualité & $32 \%$ \\
\hline Maintenir une agriculture périurbaine & $31 \%$ \\
\hline Créer de l'emploi & $28 \%$ \\
\hline Revaloriser les métiers agricoles & $24 \%$ \\
\hline Augmenter la cohésion sociale & $22 \%$ \\
\hline Adopter une alimentation plus durable & $21 \%$ \\
\hline Maintenir les commerces et les services de proximité/ lutter contre l'enclavement en milieu rural & $21 \%$ \\
\hline Limiter la dépendance alimentaire des territoires & $13 \%$ \\
\hline Éviter la disparition de certains savoir-faire & $9 \%$ \\
\hline Autre & $8 \%$ \\
\hline
\end{tabular}

Tableau 1 : Listes des principales attentes répertoriées par rapport aux circuits courts (Plusieurs réponses (3) étaient possibles parmi une liste d'items).

List of the main expected issues concerning short food chains (3 possible answers in the list)

\begin{tabular}{|c|c|c|c|c|c|}
\hline \multicolumn{2}{|c|}{ Producteurs } & \multicolumn{2}{c|}{ Consommateurs } & \multicolumn{2}{c|}{ Pouvoirs publics } \\
\hline Économique & Social & Économique & Social & Économique & Social \\
\hline $\begin{array}{c}\text { Efficacité (rapport } \\
\text { entre coût des } \\
\text { inputs et valeur de } \\
\text { la production) }\end{array}$ & $\begin{array}{c}\text { Respect d'un patri- } \\
\text { moine local (tech- } \\
\text { niques productives, } \\
\text { variétés cultivées), } \\
\text { image du métier }\end{array}$ & $\begin{array}{c}\text { Prix juste } \\
\text { Qualité (gustative } \\
\text { et sanitaire) }\end{array}$ & $\begin{array}{c}\text { Respect de normes } \\
\text { sur le travail, niveau } \\
\text { correct de rémuné- } \\
\text { ration des produc- } \\
\text { teurs et de leurs } \\
\text { employés }\end{array}$ & $\begin{array}{c}\text { Maintien et créa- } \\
\text { tion d'emplois }\end{array}$ & $\begin{array}{c}\text { Niveau correct des } \\
\text { revenus agricoles } \\
\text { et salaires, stabilité } \\
\text { des emplois créés }\end{array}$ \\
\hline
\end{tabular}

Tableau 2 : Classement des attentes vis-à-vis de la production alimentaire en circuits courts par catégorie d'acteurs Sorting of expectencies according to the type of actors

pour les familles à petit budget lorsque des innovations sociales leur facilitent l'accès à ces circuits par la moblisation des acteurs dans les territoires (Chiffoleau et Paturel, $2010^{10}$ ). Ils peuvent également offrir des occasions de valorisation touristique et culturelle dans les territoires (Scheffer, 2011) ou encore d'animation des villes : l'impact dépasse alors la sphère agricole en stimulant les services associés aux manifestations (marchés de producteurs, foires de produits locaux et traditionnels, etc.) qui sont l'occasion d'une coopération étroite entre les intérêts des producteurs et ceux des autres acteurs de l'économie locale. De la même manière, grâce à des relations renforcées avec les pairs et à l'interaction avec le consommateur, le respect de contraintes environ-

10. Chiffoleau Y., Paturel D., 2010. Short food supply chains as innovations to share quality food : evidence from case studies, communication orale au International symposium Innovation and sustainable development in agriculture and food, Montpellier, France, 28 juin-1 ${ }^{\text {er juillet } 2010 .}$ nementales peut prendre des formes qui dépassent les cadres fixés par les institutions et porter l'intérêt sur des éléments qui ne sont pas nécessairement valorisés par la labellisation : la préservation de la diversité biologique, via la culture de variétés indigènes et anciennes, peut ainsi être valorisée hors des pratiques marchandes institutionnalisées et de nouvelles pratiques culturales peuvent aussi, en retour, être valorisées au sein de la profession (Maréchal, 2008; Chiffoleau, 2009).

L'intégration de l'idée de durabilité dans les circuits courts relève également d'une construction sociale qui ne peut pas être stabilisée dans la mesure où celle-ci suppose de raisonner, à nouveau, à partir de critères de justice impliquant cette fois-ci les générations futures. Cet impératif de solidarité et de justice intergénérationnelles amène à évaluer à la fois quelle est la nature de la responsabilité des générations présentes vis-à-vis des futures et quel 
poids accorder à cette responsabilité dans les décisions présentes. A nouveau, il s'agit bien de processus démocratiques indispensables. Les circuits courts participent à ces processus en permettant l'implication des différents acteurs concernés et un apprentissage mutuel pour savoir comment réaliser les objectifs multidimensionnels finalement assignés à la production alimentaire : nous avons étudié, par exemple, la mise en place d'une boutique de producteurs dans un quartier défavorisé d'une ville du Languedoc-Roussillon, visant à la fois à soutenir des producteurs en difficulté et à être accessible aux familles à petit budget du quartier. Répondre à l'enjeu d'une double solidarité a supposé l'animation d'un débat avec d'autres catégories d'acteurs pour faire connaître aux uns les problèmes du secteur agricole et aux autres la réalité des dépenses des ménages pauvres ${ }^{11}$, qui a finalement permis une mixité sociale, de producteurs comme de consommateurs et la fixation de deux niveaux de prix « justes », fondant ainsi une innovation territoriale plus favorable au projet de développement durable (Chiffoleau et Paturel, 2010). Ce type d'initiative appelle alors à une réflexion sur les nouveaux indicateurs de bien-être nécessaires pour évaluer la performance des circuits courts au sein d'un système global d'alimentation durable : quels sont les critères pertinents et à quelle échelle penser et évaluer ce système alimentaire? À nouveau, la stabilité est incertaine : les échelles d'évaluation (les différents niveaux de territoires, pays, monde) dépendent des critères retenus et inversement. La rationalisation des systèmes de décision est donc incomplète et suppose un débat permanent et des efforts d'innovation pour concevoir les procédures collectives les plus efficaces pour faire vivre ce débat.

\section{« Empowerment ", démocratie locale et innovations territoriales}

Appréhendé dans sa version la plus simple comme une " expansion de la liberté de choix et d'action » (Banque Mondiale, 2002) ou comme renforcement de la « capacité d'une personne à faire des choix $[\ldots]$ et à $[$ les] transformer en actions et résultats

11. Souvent contraintes à acheter au détail et près de chez eux, du fait de manque de ressources pour acheter en gros, stocker, cuisiner ou encore se déplacer, les familles pauvres consacraient en 2009 près de $20 \%$ de leur budget à l'alimentation contre $14 \%$ pour la moyenne des Français, d'après l'INSEE. désirés » (Alsop et Heinsohn, 2005), l'« empowerment » peut être directement rattaché à l'approche en termes de capabilités proposée par Sen (Narayan, 2006; Alkire, 2008). Il s'agit en effet de s'intéresser à l'équité non pas sous l'angle de ce que les gens réalisent ou non mais à partir de l'ensemble des possibilités de choix qui s'ouvrent à eux et leur permettent d'agir dans les différentes sphères de la vie sociale.

On trouve ici deux idées fortes :

- La pauvreté doit s'analyser avant tout comme un processus, ce qui conduit à s'intéresser à la vulnérabilité ou à la fragilité des individus tout autant qu'à leur fragilisation. L'empowerment ${ }^{12}$ se pense alors comme un processus inverse au cours duquel les agents sont sécurisés et renforcés dans leur capacité à faire des choix et à les réaliser.

- La fragilisation relève de manques de capacités dans différents domaines et l'empowerment passe alors par un renforcement multidimensionnel de ces capacités.

Partant de ces bases, le renforcement des capacités peut se décliner en plusieurs modalités : le pouvoir de réaliser des choix (pouvoir de), le pouvoir d'agir avec les autres (pouvoir avec), le pouvoir intérieur ou estime de soi, le pouvoir sur les autres (Csazar, 2004). La dernière dimension est la plus rarement explorée dans la littérature économique (Prévost, 2010; Palier et Prévost, 2005, 2007) et nous n'entrerons pas dans les débats que cela occasionne. On peut toutefois partir de là pour soulever que l'empowerment passe par une réduction des dominations subies, autrement dit par la mise en place de relations moins asymétriques et moins hiérarchiques. On peut considérer que cette démarche est même à la base des circuits courts qui consistent souvent, pour les producteurs, à s'émanciper de relations marchandes asymétriques entretenues avec les intermédiaires qui contrôlent et régulent les marchés agroalimentaires : grossistes, grandes et moyennes surfaces (GMS), industries de transformation, etc. En cela, les circuits courts s'appuient sur une démarche proche de celle du commerce équitable (Ferraton et Prévost, 2010) et visent à mettre sur pied des relations d'échange moins intermédiées et plus proches d'une relation de clientèle que d'une relation marchande pure et simple.

12. Malgré l'anglicisme a priori, nous continuons à utiliser « empowerment » dans le texte car ce terme est aujourd'hui diffusé largement dans les medias et discours politiques. 
Néanmoins, cette personnalisation de la relation commerciale n'est pas nécessairement à la base des circuits courts dont la diversité supporte des formes d'échange ou de coordination aux fondements différents (Holloway et al., 2007 ; Vincq et al., 2010). Reste l'objectif essentiel et commun aux différentes formes de circuits courts : limiter le nombre d'intermédiaires pour permettre une meilleure captation de la valeur ajoutée par les producteurs, même si les difficultés du système d'activités imposé par la vente en circuit court peuvent néanmoins limiter le revenu qui peut en être tiré, du moins au démarrage et si ce revenu est ramené à l'heure de travail réellement passé (Capt et Chiffoleau, 2011).

Cette dimension du renforcement des producteurs, si elle est essentielle et motive l'intérêt des pouvoirs publics pour les circuits courts, doit de plus être pensée à la fois dans son articulation avec les différentes dimensions de cet empowerment et, surtout, en articulation avec l'empowerment des consommateurs ainsi qu'à travers l'impact de ces interactions sur la manière de concevoir ensuite une alimentation durable.

\section{Renforcer les producteurs}

L'insertion des producteurs dans de nouvelles relations d'échange s'appuie sur des motivations qui peuvent dépasser l'argument purement financier, comme l'avaient déjà montré différents travaux sur la vente directe notamment (Le Caro et Daniel, 2007). Plus largement, la prise en compte des pratiques et des réseaux développés autour des circuits courts avec 0 ou 1 intermédiaire permettent de distinguer par exemple cinq profils contrastés de producteurs en Languedoc-Roussillon (Pellequer et Chiffoleau, 2009) :

- les «pragmatiques », qui combinent circuits longs et circuits courts dans une optique de rentabilité et étaient ou deviennent les piliers de l'approvisionnement des villes et des grandes et moyennes surfaces du périurbain;

- les «innovateurs", qui s'engagent fortement, financièrement et en temps, et multiplient leurs activités à un rythme soutenu pour s'inscrire dans les circuits courts, en proposant aux citadins des services souvent attendus autour de ces circuits, tels que l'accueil ou des activités de loisirs;
- les « hédonistes efficaces ", qui cherchent à allier rentabilité et qualité de vie, figures clés des marchés de plein vent des villes;

- les «spécialistes engagés », se consacrant à la la vente directe, qui envisagent la production en circuit court comme une activité leur permettant d'acquérir un certain revenu mais aussi, voire surtout, de construire un engagement citoyen, à travers les systèmes tels que les AMAP notamment;

- les «idéalistes ", qui cherchent un « retour à la terre » et misent sur une production de qualité, essaient de vendre en local mais finalement, ne sont pas très intéressés par l'activité de commercialisation.

Il ne s'agit pas de faire de ces types de producteurs des formes figées et sans lien : c'est la pluralité des logiques qui caractérise les actions économiques et qui d'ailleurs en rend difficile la modélisation. Néanmoins, les différentes aspirations exprimées renvoient nécessairement à des formes d'empowerment distinctes.

Le pouvoir de englobe ainsi les gains en autonomie que peuvent permettre les circuits courts par la sortie des relations asymétriques caractérisant a priori les circuits traditionnels. La capacité à réaliser ses choix, quels qu'ils soient, suppose néanmoins une confrontation régulière à l'épreuve marchande ce qui implique une amélioration des capacités des producteurs à faire face à l'ensemble des impératifs qui régulent l'épreuve marchande et en particulier la maîtrise des coûts. L'évaluation de la performance des circuits courts est l'objet d'attentions croissantes de la part des pouvoirs publics ${ }^{13}$ car elle est bien sûr un enjeu majeur mais qui soulève de très nombreuses questions dont les solutions échappent en partie aux exigences de la comptabilité (Capt et Chiffoleau, 2011). En particulier, l'autonomie gagnée dans les circuits courts se traduit par une montée en charge des responsabilités liées à la nécessité d'assumer des fonctions jusque là prises en charge par les intermédiaires. Ces responsabilités se traduisent nécessairement par des niveaux d'activité et de compétences nouveaux qui modifient le métier des producteurs. Outre que ces charges sont difficiles à évaluer d'un point de vue comp-

13. Le manque de références technico-économiques sur les exploitations en circuits courts a été l'un des premiers freins à leur développement évoqués par le groupe de travail formé par le MAAP en 2009, motivant la commande d'une étude à l'INRA (Capt et Chiffoleau, 2011) et l'intégration de nouvelles questions sur ce thème dans le Recensement Agricole de 2010. 
table, elles supposent également de s'interroger sur la manière dont les producteurs peuvent acquérir les compétences exigées.

Ici, le pouvoir avec peut jouer un rôle important grâce à l'insertion dans des réseaux sociaux porteurs d'un capital social permettant d'accéder à un ensemble de ressources qu'il n'était jusque là pas nécessaire de mobiliser. La nouvelle sociologie économique permet d'analyser la manière dont les circuits courts favorisent l'émergence de nouvelles relations entre producteurs, que ce soit via les marchés ou les AMAP, qui entraînent une augmentation de la performance économique en permettant apprentissage et collaboration (Chiffoleau, 2009). Cette analyse rejoint les nombreux travaux développés sur les systèmes alimentaires territorialisés (Syal) qui montrent en quoi les espaces marchands agricoles, a priori lieux de concurrence, sont aussi des lieux de coopération et d'échange entre producteurs (Muchnik, De Sainte-Marie, 2010). Elle la complète en précisant la forme de pouvoir qui en est issue, alors que cette notion reste peu approfondie dans les travaux sur les Syal producteurs (Muchnik, De Sainte-Marie, 2010)

L'amélioration de l'estime de soi (pouvoir intérieur) est une dimension également essentielle des processus d'empowerment et tout particulièrement en agriculture où l'image des producteurs a souffert d'une série de crises liées à l'adoption de pratiques peu respectueuses non seulement de l'environnement mais aussi des produits eux-mêmes (Joly et Paradeise, 2003). Si l'estime de soi se joue en grande partie dans la relation aux pairs et favorise l'action collective, elle se joue aussi dans le rapport aux consommateurs qui sont porteurs de ces attentes, dans les villes en particulier où la recherche de rapprochement urbain-rural s'inscrit dans la quête de sens de la société hypermoderne, comme le montrent certains de nos travaux (Chiffoleau, 2009; Chiffoleau et Dreyfus, 2011).

Nos travaux sur les AMAP et surtout sur certaines formes plus souples qui en sont issues ${ }^{14}$ montrent en quoi ces systèmes forment un cas exemplaire de projet d'alimentation pour les territoires urbains où le dialogue entre producteurs et consommateurs

14. Le Languedoc-Roussillon, terrain principal de nos analyses, montre en effet un développement important de systèmes de vente par paniers (de légumes) ou caissettes (de viande), aujourd'hui plus nombreux que les AMAP dans cette région et ne fonctionnant pas par abonnement à l'avance; ces systèmes témoignent néanmoins d'un noyau de clients fidèles. permet de décliner plusieurs formes d'empowerment : le pouvoir avec dans le cadre d'une relation de clientèle qui implique une personnalisation des échanges favorable à des liens de réciprocité; c'est ce lien d'échange spécifique qui génère ensuite un pouvoir de grâce à la stabilisation des débouchés et des prix de vente qui réduit les incertitudes pour les producteurs; il est également à l'origine d'un pouvoir intérieur lié à l'amélioration de la perception du travail agricole via un ensemble de normes sur la qualité des produits et des pratiques, formalisées ou non. Cette valorisation du métier prend donc un sens économique grâce à une meilleure valorisation des produits porteurs de ces compétences spécifiques reconnues : intégration à la fois de la qualité intrinsèque des produits, mais aussi intégration des externalités positives de cette agriculture ${ }^{15}$. D'une certaine manière, les consommateurs décident de prendre à leur charge la rémunération de ces externalités en favorisant le maintien d'une agriculture que le marché condamnerait, notamment dans les espaces périurbains dominés par la spéculation foncière, alors qu'elle est socialement utile, tout particulièrement dans ces espaces qui favorisent la rencontre ville-campagne. Le prix supplémentaire payé éventuellement ou, plus souvent, perçu, par les consommateurs est une reconnaissance de cette utilité sociale. Elle suppose toutefois un dialogue constant entre consommateurs et producteurs dans la mesure où l'utilité sociale n'est pas arbitrée par la force objective d'un quelconque mécanisme d'évaluation marchande : elle se construit dans des interactions qui permettent l'établissement de préférences individuelles et collectives qui seront valorisées à la fois par chacun et collectivement (Chiffoleau et Dreyfus, 2011).

\section{Construire une alimentation durable par les innovations territoriales}

Le rattachement d'une utilité sociale à l'agriculture s'appuie sur l'ensemble des externalités géné-

15. Néanmoins, la spécificité des AMAP ne doit pas masquer à la fois les éventuels échecs, comme par exemple le sentiment d'une perte d'autonomie par les producteurs dans l'établissement d'un contrat les liant au groupe de consommateurs (Dufour et al., 2010). Ce sentiment est d'ailleurs contradictoire : la relation de clientèle qui est à la base des avantages liés aux AMAP se caractérise justement par son inscription dans le temps et la constitution d'un ensemble de règles relationnelles qui, pour assurer la pérennité, limite nécessairement les libertés individuelles. 
rées par la production agricole. L'alimentation est ainsi conçue comme un phénomène social non pas seulement parce qu'elle est déterminée par des facteurs sociaux tout autant qu'économiques, mais bien parce qu'elle met en jeu des choix collectifs en matière de développement. Si ces enjeux ont longtemps été confinés à la fois à une approche d'une part purement économique et technique et d'autre part nationale, ils sont aujourd'hui conçus à l'échelle territoriale : le discours sur la relocalisation de la production agricole et sur l'autonomie alimentaire des territoires s'inscrit dans un processus complexe à la fois de réappropriation de la question alimentaire mais aussi de territorialisation des politiques publiques (Amemiya, 2011). L'évolution vers une régulation territoriale de l'activité agricole, notamment via les évolutions de la Politique Agricole Commune (Berriet-Solliec et al., 2009; Trouvé, 2009), n'est qu'une partie d'une dynamique de territorialisation des stratégies de développement: l'analyse des innovations sociales liées aux circuits courts s'inscrit à la fois dans la spécificité d'une « grande transformation de l'agriculture » (Boyer et Allaire, 1995) et dans les problématiques plus générales du développement local (Marsden et al., 1999). Notamment, si le désengagement de l'État entraîne des difficultés à financer localement les politiques de développement et pose des problèmes d'inégalités entre les territoires, il ouvre également des espaces pour les initiatives et les innovations sociales dans une dynamique où les populations peuvent se ressaisir des questions collectives et impulser du développement "par le bas », comme en témoignent les initiatives observées dans le cadre du Réseau rural français.

Les circuits courts offrent un support à ces dynamiques et illustrent à leur manière certaines ambiguïtés des liens entre désengagement de l'État et initiatives locales. Nous prendrons ici le cas de la restauration collective dans les villes que l'on peut considérer au cœur des questions liées à une alimentation durable. A émergé au cours des dernières années l'idée d'une restauration collective responsable, incarnée par les travaux conjoints de la Fondation Nicolas Hulot et des CIVAM ${ }^{16}$. L'idée est de soutenir « une dynamique citoyenne, de la terre

16. Centres d'Initiatives pour Valoriser l'Agriculture et le Milieu rural. à l'assiette ${ }^{17}$ », dans une perspective où l'alimentation est pensée comme un système global incluant l'ensemble de la chaîne sociale qui va de l'activité agricole jusqu'à la consommation finale de sa production. Il s'agit alors de :

- restaurer la qualité environnementale;

- relocaliser les systèmes alimentaires;

- former au goût, à la qualité, à la santé;

- réinstaller et sécuriser les débouchés pour les producteurs locaux.

L'attente d'une alimentation de qualité dans les cantines scolaires a d'abord été formulée à travers la demande de produits biologiques par des consommateurs ou élus urbains sans que la référence à une production locale soit évoquée, notamment du fait de leur méconnaissance voire ignorance des réalités du secteur agricole. L'introduction de la proximité dans les attentes a été favorisée par la diffusion médiatique des difficultés du monde agricole et la montée en puissance des circuits courts : ce qui était en cours dans les échanges marchands impliquant des individus a pu être transposé dans le cadre d'une problématisation collective. Le lien avec les circuits courts a justifié la mise en place, au sein du Réseau rural français, d'un axe de travail spécifique.

Le passage d'une restauration collective assurée par des groupes industriels disposant de structures productives efficientes et bénéficiant de rendements d'échelle, à une restauration collective s'appuyant sur des producteurs locaux pose de nombreux problèmes. Un premier problème est d'ordre institutionnel et réglementaire : le code des marchés publics ne permet pas pour l'instant d'intégrer dans le cahier des charges des références à la localité des produits. Cela suppose alors un mouvement d'adaptation/contournement qui ne peut s'appuyer que sur des règles informelles : le capital social joue ici un rôle important à la fois comme cadre de discussion à l'élaboration de ces règles (amont) et, ensuite, au respect de ces règles (aval). Cela permet également une réflexion citoyenne sur le sens de la réglementation et son adéquation aux besoins collectifs. Les contraintes réglementaires peuvent ainsi apparaître comme des leviers d'innovations territoriales (Sonnino, 2010) en modifiant les liens entre les acteurs (responsables des collectivités et de la

17. Guide disponible en ligne [http://www.fondation-nicolas-hulot.org/actions/ alimentation-responsable/pour-une-restauration-collective-responsable]. 
restauration collective, fournisseurs, producteurs). On entre ici dans le cadre expliqué en introduction, à savoir l'analyse des effets d'entraînement entre innovations.

Ces innovations sont toutefois dépendantes des contextes locaux et des jeux d'acteurs. Il est ainsi intéressant de suivre les dynamiques informelles à l'œuvre dans cette adaptation aux contraintes formelles et de voir comment elles peuvent parvenir éventuellement à une institutionnalisation qui certes permet de dépasser les contraintes réglementaires mais implique également une rationalisation et une homogénéisation qui à leur tour viendront modifier les équilibres informels trouvés dans une construction conjointe par les acteurs (Le Velly et al., 2010).

Le problème est ensuite celui d'une adaptation de l'offre aux besoins de la restauration collective, en particulier la fourniture de matières premières prêtes à l'emploi. Il s'agit d'ailleurs ici d'une contrainte liée également au manque de moyens humains et de compétences dans les cantines elles-mêmes (possibilité ou pas de traiter les produits bruts), mais aussi au manque de motivation du personnel, souvent exclu du dialogue autour des nouveaux enjeux associés et ne disposant pas des ressources nécessaires à une participation critique (Friedberg, 1987). Les besoins en matière d'équipements collectifs sont ainsi essentiels et ont une influence déterminante sur les coûts et donc, in fine, sur le niveau des prix des repas qui peut être une contrainte majeure dans l'adoption, par les collectivités, de nouvelles logiques de restauration collective. Les coûts de mise en œuvre d'équipements collectifs posent à leur tour une difficulté à laquelle peuvent répondre des innovations, comme le montrent des initiatives autour de SCIC $^{18}$ : ces « coopératives de territoire » apparaissent comme une forme particulièrement innovante en matière agricole pour repenser non seulement les modes de financement mais aussi les modes d'action collective des producteurs et l'implication des différentes parties prenantes du développement local, dont le personnel des cantines (Thomas, 2009).

18. Société Coopérative d'Intérêt Collectif.

\section{Conclusion : Vers une gouver- NANCE ALIMENTAIRE LOCALE}

Différentes dynamiques autour de l'alimentation, du national au local, conduisent aujourd'hui à l'émergence d'une gouvernance alimentaire territoriale reposant sur de nouvelles modalités d'implication des acteurs. Cette gouvernance suppose la mise en place de procédures démocratiques permettant la formation et l'expression de nouvelles attentes qui relèvent de la prise en main d'une destinée collective. Les circuits courts peuvent jouer un rôle déterminant dans ces évolutions récentes de par leur capacité à reformuler les liens marchands dans le sens d'une coopération accrue non seulement entre producteurs mais aussi entre producteurs et consommateurs, entre consommateurs, entre acteurs d'un même territoire ou d'une même région. De nombreuses villes renouvellent ainsi leurs liens avec les territoires qui les approvisionnent, ce qui construit de nouveaux espaces sociaux et géographiques de concertation ${ }^{19}$. La reformulation marchande qu'y s'y joue ne prend toutefois son sens qu'à partir du moment où elle stimule en retour des innovations sociales influençant l'ensemble des perspectives du développement local durable. Dans une dynamique de réforme de la PAC où le marché est censé orienter de manière plus efficace les productions et renforcer les processus de spécialisations régionales (Barkaoui et al., 2009), l'idée que les populations se ressaisissent des enjeux alimentaires permet d'ouvrir le champ des possibles en matière de développement rural et péri-urbain. En effet, la demande d'une agriculture de proximité est a priori contradictoire avec les tendances liées à l'efficacité économique d'une agriculture industrialisée et orientée par les marchés mondiaux. Comment peut alors évoluer la cohabitation de plusieurs systèmes agro-alimentaires et quel rôle peuvent jouer les villes pour construire et défendre des systèmes alimentaires territorialisés?

De nombreuses questions subsistent auxquelles la recherche doit répondre. Il s'agit en effet de vérifier de nombreuses hypothèses concernant les vertus supposées des circuits courts. En premier lieu, la viabilité économique des exploitations en circuits courts reste à évaluer. En dehors des difficultés

19. Le lecteur trouvera notamment des exemples éclairants à travers les travaux de l'association Terres en Villes : [http://www.terresenville.org]. 
propres à l'évaluation économique et comptable de ces exploitations, l'une des questions essentielles reste de savoir comment définir leur performance. Si l'on accepte l'idée suivant laquelle les circuits courts génèrent de nombreuses externalités positives, dans quelle mesure doit-on prendre en compte ces externalités dans l'évaluation de la performance? On l'a dit, le fait que beaucoup de consommateurs sont prêts à payer plus cher les produits issus de la production locale, comme pour les produits biologiques ou issus du commerce équitable, est d'une certaine manière une forme d'évaluation, par les consommateurs, de ces externalités positives. L'un des enjeux majeurs est alors d'identifier les difficultés spécifiques des circuits courts et les leviers qui peuvent être actionnés pour les dépasser. Compter sur la seule bonne volonté des consommateurs relèverait d'une logique purement marchande : le prix supplémentaire illustrerait les principes de la demande tels qu'ils sont formulés par la théorie standard, le sur-prix étant alors un consentement à payer pour accéder à une alimentation durable respectant un ensemble de critères définis par les acteurs euxmêmes. Une telle logique exclut nécessairement une part importante de la population, sur-responsabilise les consommateurs et dédouane l'État au regard du projet d'alimentation durable (Figuié et Bricas, 2009). Surtout, cette approche est fermée sur une évaluation à court terme : les effets de seuil liés aux rendements d'échelle doivent être pris en compte pour évaluer quel serait, à terme, le surcoût des circuits courts par rapport aux circuits traditionnels. Ce surcoût, surtout, devrait être comparé à la fois à celui des subventions dont bénéficie l'agriculture insérée dans les logiques conventionnelles et aux externalités positives que les territoires et les villes pourraient retirer de ces nouveaux facteurs de développement intégré que sont les circuits courts. Il convient alors de poser publiquement le débat sur ce qui doit être mesuré et sur la façon de le mesurer.

Si l'on considère les territoires comme des systèmes d'interactions complexes, c'est l'ensemble de ces systèmes dont il convient d'analyser la performance. Aux effets d'entraînement traditionnellement étudiés en économie, dont la mesure n'est elle-même pas toujours aisée (Léon et Surry, 2009), s'ajoutent des effets d'entraînement au niveau des innovations dont la mesure semble encore plus problématique. Se posent alors plus de questions que la recherche n'est capable de fournir de réponses dans l'immédiat.

On en revient, finalement, à l'importance des principes démocratiques dans les procédures d'évaluation. Les débats actuels sur les nouveaux indicateurs de richesse, de bien-être et de développement sont l'occasion de mettre en place des dispositifs d'évaluation participative qui permettent de mettre en relief les attentes individuelles et collectives des populations qui sont à la fois actrices et bénéficiaires du développement local (Renault et al., 201020). C'est bien l'un des principes fondamentaux de la démocratie que de consulter les populations sur les décisions qui les affectent (Fleurbaey, 2006). Les circuits courts sont une bonne entrée pour reposer la question démocratique au niveau local et analyser comment l'économique, le social et le politique se conjuguent pour produire la durabilité de systèmes alimentaires en mutation, en réponse aux nouveaux enjeux des villes en particulier.

\section{Bibliographie}

Akerlofgener-bibliography G., 1970. The Market for Lemons: Qualitative Uncertainty and the Market Mechanism, Quarterly Journal of Economics, vol. 84, n 70, p. 488500.

AlkiRe S., 2008. Concepts and measures of agency, OPHI, Working Papers, n 9, 23 p.

Alsop R., Heinsohn N., 2005. Measuring Empowerment in Practice: Structuring Analysis and Framing Indicators, The World Bank, Washignton, Policy Research Working Paper, $n^{\circ} 3510$, February 2005, 123 p.

Amemiya H. (éd.), 2011. Du teikei aux AMAP, le renouveau de la vente directe de produits fermiers locaux, Rennes, PUR, $350 \mathrm{p}$.

Amemiya H., Bénézech D., Renault M., 2008. Les circuits courts : un monde de commercialisation interpersonnel?, dans Maréchal G., Les circuits courts alimentaires, Paris, Educagri, p. 113-123.

Anderson E., 2003. Sen, ethics and democracy, Feminist Economics, vol. IX, n 2-3, p. 239-261.

Banque Mondiale, 2002. Empowerment and Poverty Reduction: A Sourcebook, World Bank, Washington, 280 p.

Barkaoui A., Daniel K., Butault J.-P., 2009. Impact territorial de la réforme de la PAC de 2003. Enjeux du découplage, dans Aubert F., Piveteau V., Schmitt B., Politiques agricoles et territoires, Versailles, Quae, p. 119-140.

20. Renault M., Cartieaux C., Jaouen C., Meriot P., 2010. La construction participative d'indicateurs de bien-être, retour sur une expérience, communication orale au $1^{\text {er }}$ Congrès de l'Association Française d'Economie Politique, Lille, 9-10 décembre 2010. 
Benicourt E., 2007. Amartya Sen, un bilan critique, Cahiers d'économie politique, $\mathrm{n}^{\circ}$ 52, p. 57-81.

Berriet-Solec M., Le Roy A., Trouve A., 2009. Territorialiser la politique agricole pour plus de cohésion, Économie rurale, $n^{\circ} 313-314$, p. 129-146.

Bouchard M., 2006. L'innovation sociale en économie sociale, dans Harrison D., Klein J.-L. (eds), L'innovation sociale, émergence et effets sur la transformation des sociétés, Québec, Presses de l'Université du Québec, p. 121-138.

Boyer R., Allaire G., 1995. La grande transformation en agriculture, Paris, Economica, 445 p.

Capt D., Chiffoleau Y. (coord.), 2011. Élaboration d'un réféntiel technico-économique dans le domaine des circuits courts de commercialisation en agriculture, Montpellier/Dijon, INRA/AgroSupDijon, Rapport de recherche pour le MAAP, non paginé.

Chiffoleau Y., 2009. From politics to co-operation : the dynamics of embeddedness in alternative food supply chains, Sociologia ruralis, vol. 49, n³, p. 218-235.

Chiffoleau Y., Dreyfus F., 2011 . Hardy outdoor breeding. Innovation networks for functional integrity in agri-food processing systems, Haut-Languedoc, France, dans Kamilli T., Hubert B., Tourrand J.F. (eds), A paradigm shift in lifestock management: from resource sufficiency to functional integrity, Lirac, Cardère Editeur, p. 161-176.

Chiffoleau Y., Laporte C., 2004. La formation des prix : le marché des vins de Bourgogne, Revue Française de Sociologie, vol. 45, n 4, p. 663-680.

Codron J.-M., Sirieix L., Reardon T., 2006. Social and environmental attributes in an emerging mass market : challenges of signaling and consumer perception, with European illustrations, Agriculture and Human Values, vol.23, $n^{\circ} 3$, p. 283-297.

Csazar F., 2004. Understanding the Concept of Power, dans Alsop R. (ed.), Power, Rights and Poverty : Concepts and connections, Washington, The World Bank, p. 137-147.

De Munck J., 2008. Qu'est-ce qu'une capacité?, dans De Munck J., Zimmermann B. (eds), La liberté au prisme des capacités. Sen au-delà du libéralisme, Raisons pratiques, Paris, École des hautes études en sciences sociales (EHESS), p. 21-49.

De Munck J., Zimmermann B. (eds) 2008. La liberté au prisme des capacités. Sen au-delà du libéralisme, Paris, École des hautes études en sciences sociales (EHESS), Raisons pratiques, $334 \mathrm{p}$.

Dubuisson-Quellier S., Le Velly R., 2008. Les circuits courts, entre alternative et hybridation, dans MARÉchAL G. (coord.), Les circuits courts alimentaires, bien manger dans les territoires, Dijon, Educagri, p. 105-112.

Dubuisson-Quellier S., Lamine C., 2004. Faire le marché autrement. L'abonnement à un panier de fruits et légumes comme forme d'engagement politique des consommateurs, Sciences de la Société, nº 62, p. 145-168.

Dufour A., Lanciano E., Herault C., Pennec N., 2010. L'herbe est-elle plus verte dans le panier? Satisfaction au travail et intégration professionnelle de maraîchers qui commercialisent sous forme de paniers, dans Traversac J.-B. (éd.), Circuits courts. Contribution au développement régional, Dijon, Educagri, p. 71-85.

Ferraton C., Prevost B., 2010. Les ambiguïtés du commerce équitable : l'ESS peut-elle se passer d'une théorie de la justice?, communication orale aux Xe rencontres $d u$ Réseau Inter-Universitaire de l'Économie Sociale et Solidaire (RIUESS), Luxembourg.

Figuié M., Bricas N., 2009. Équité internationale. La surresponsabilisation des consommateurs, Courrier de la Planète, $\mathrm{n}^{\circ} 87$, p. 41.

Fleurbaey M., 2006. Capitalisme ou démocratie? L'alternative du xxie siècle, Paris, Grasset, 214 p.

Friedberg E., 1987. L'analyse sociologique des organisations, Pour, $\mathrm{n}^{\circ} 28$

Granovetter M.S., 1985. Economic action and social structure : the problem of embeddedness, American Journal of Sociology, vol. 91, n³, p. 481-510.

Granovetter M.S., 2000. Le marché autrement. Paris, Desclée de Bouwer, $240 \mathrm{p}$.

Harrison D., Klein J.-L. (eds), 2006. L'innovation sociale, émergence et effets sur la transformation des sociétés, Québec, Presses de l'Université du Québec, 465 p.

Harrison D., Vezina M., 2006. L'innovation sociale, une introduction, Annals of Public and Cooperative Economics, vol. $77, \mathrm{n}^{\circ} 2$, p. 129-139.

Hinrichs C., 2000. Embeddedness and local food systems : notes on two types of direct agricultural market, Journal of Rural Studies, ${ }^{\circ}$ 16, p. 295-303.

Joly P.-B., Paradeise C., 2003. Agriculture et alimentation : nouveaux problèmes, nouvelles questions. Introduction, Sociologie du Travail, $\mathrm{n}^{\circ} 45$, p. 1-8.

Karpik L., 1989. L'économie de la qualité, Revue française de sociologie, vol. $30, \mathrm{n}^{\circ} 2$, p. 187-210.

LAmine C., 2005. Settling shared uncertainties : local partnerships between producers and consumers, Sociologia Ruralis, Vol. 45, n 4 , p. 324-345.

Laville J.-L., 2006. Eléments pour l'analyse du changement social démocratique, in L'innovation sociale, émergence et effets sur la transformation des sociétés, dans Harrison D., KLein J.-L (eds), Québec, Presses de l'Université du Québec, p. 89-120.

Le Caro Y., Daniel R., 2007. Les motivations des agriculteurs d'après une enquête auprès de vendeurs directs en Bretagne, dans AmemiYa H. (éd.), L'agriculture participative, dynamiques bretonnes de la vente directe, Rennes, Presses Universitaires de Bretagne, p. 95-124.

Leon Y., Surry Y., 2009. Les effets d'entraînement du complexe agroalimentaire au niveau local, dans Aubert F., Piveteau V., Sснмітт B. (ed.), Politiques agricoles et territoires, Versailles, Editions Quae, p. 21-48.

Le Velly R., Bottois V., Brechet J.-P., Chazoule C., Cornee M., Emin S., Praly C., Schieb-Bienfait N., 2010. Comment se fait la rencontre entre offres et demandes locales pour la restauration collective? Premiers enseignements, dans Traversac J.-B. (éd.), Circuits courts. Contribution au développement régional, Dijon, Educagri, p. 187-194. 
Lipovetsky C., Charles S., 2004. Les temps hypermodernes, Paris, Grasset, 125 p.

Marchesin P., 2004. Démocratie et développement, Revue Tiers monde, vol. 45, n 179, p. 487-513.

Marechal G. (coord.), 2008. Les circuits courts alimentaires, bien manger dans les territoires, Dijon, Educagri, 213 p.

Marsden T., Murdoch J., Morgan K., 1999. Sustainable agriculture, food supply chains and regional development, International Planning Studies, vol. 4, n³ 3, p. 295-301.

Martins N., 2007. Ethics, Ontology and Capabilities, Review of Political Economy, vol. XIX, n ${ }^{\circ}$ 1, p. 37-53.

Merle A., Piotrowski M., 2012. Consommer des produits alimentaires locaux : comment et pourquoi? Décisions marketing, $\mathrm{n}^{\circ} 67$, à paraître.

Muchnik J., De Sainte-Marie C., (coord.), 2010. Le temps des SYAL, techniques, vivres et territoires, Paris, Quae, 320 p.

Narayan D. (éd.), 2006. Measuring Empowerment, Cross-Disciplinary Perspectives, Oxford, Oxford University press, 496 p.

Nussbaum M. C., 2001. Adaptative Preferences and Women Options, Economics and Philosophy, n ${ }^{\circ}$ 17, p. 67-88.

Nussbaum M.C., 2003. Capabilities as Fundamental Entitlements : Sen and Social Justice, Feminist Economics, vol. IX, $\mathrm{n}^{\circ} 2-3$, p. 33-58.

Nussbaumer J., Moulaert F., 2008. La logique sociale du développement local, Québec, Presses de l'Université du Québec, 153 p.

Palier J., Prévost B., 2005. Le développement social : nouveau discours et idéologie de la Banque Mondiale, Economie Appliquée, vol. LX, n 4, p. 27-50.

Palier J., Prévost B., 2007. Vulnérabilité et gestion des risques : potentialités et limites de la microfinance. L'exemple de l'Inde du Sud, Mondes en développement, n 138, p. 103-118.

Pellequer D., Chiffoleau Y., 2009. Construire un observatoire territorial pour piloter les circuits courts, Les Cahiers de l'Observatoire Coxinel, $\mathrm{n}^{\circ} 3$, septembre 2009, [en ligne] ULR : [http://www.psdr-coxinel.fr].

Peтtrt P., 2004. Penser en société, essai de métaphysique sociale et de méthodologie, Paris, PUF, 160 p.

Prévost B., 2009. Sen, la démocratie et le marché, Revue Tiers Monde, $\mathrm{n}^{\circ} 198$, p. 269-285.

Prévost B., 2010. Lève-toi et marche! Les injonctions de l'empowerment, Economie et Institutions, n 12, p. 19-44.

Prévost B., 2011. Circuits courts et innovations territoriales : de l'échange à la démocratie, Chantiers Politiques, $n^{\circ} 9$, p. 46-56.
Putnam H., 2002. The Collapse of the Fact/Value Dichotomy and Other Essays, Cambridge, Harvard University Press, 190 p.

Redlingshöfer B., 2006. Vers une alimentation durable? Ce qu'enseigne la littérature scientifique, Le courrier de l'environnement de l'INRA, n 53, p. 83-102.

Richez-Battesti N., Vallade D., 2009. Économie sociale et solidaire et innovation sociale : Premières observations sur un incubateur dédié en Languedoc-Roussillon, Innovations, 2009/2, n³0, p. 41-69.

Robeyns I., 2003. Sen's Capability Approach and Gender Inequality : Selecting Relevant Capabilities, Feminist Economics, vol. IX, p. 61-92.

Robeyns I., 2005. The Capability Approach : A Theoretical Survey, Journal of Human Development, vol. VI, n 1, p. 93-114.

SchefFer S., 2011. La dimension touristique des marchés, une forme particulière de renouveau, dans TraversaC J.-B. (éd.), Les circuits courts, contribution au développement régional, Dijon, Educagri, p. 129-152.

SEN A., 2003. Un nouveau modèle économique, Paris, Odile Jacob, $480 \mathrm{p}$.

Sen A., 2006. La démocratie des autres, Paris, Payot, 86 p.

Sonnino R., 2010. Escaping the Local Trap: Insights on Relocalization from School Food Reform. Journal of Environmental Policy and Planning, vol. 12, $\mathrm{n}^{\circ}$ 1, p. 23-40.

Thomas F., 2009. SCIC et agriculture : le temps des défricheurs, RECMA - Revue internationale de l'économie sociale, $\mathrm{n}^{\circ} 310$, p. 17-30.

Trouvé A., 2009. Les régions, porteuses de nouveaux compromis pour l'agriculture?, Revue de la régulation, [En ligne], $\mathrm{n}^{\circ} 5,1^{\text {er }}$ semestre 2009, mis en ligne le 16 juin 2009, URL: [http://regulation.revues.org/index $7550 . h \mathrm{tml}]$.

Vince J.-L., Mondy B., Fontorbes J.-P., 2010. La construction de la qualité fiable dans les réseaux alimentaires de proximité, Economie rurale, n³18-319, p. 5-19.

Walsh V., 2000. Smith after Sen, Review of Political Economy, vol. XII, n 1 , p. 5-25.

Walsh V., 2003. Sen after Putnam, Review of Political Economy, vol. XV, n 3, p. 315-394.

White H. C., 1992. Identity and control: a structural theory of social action, Princeton, Princeton University Press, 448 p.

White H. C., 2002. Markets from networks. Socioeconomic models of production, Princeton, Princeton University Press, $384 \mathrm{p}$. 